\title{
Efficacy of a smartphone-based coaching program for addiction prevention among apprentices: study protocol of a cluster- randomised controlled trial
}

\author{
Severin Haug ${ }^{*}$, Raquel Paz Castro, Andreas Wenger and Michael P. Schaub
}

\begin{abstract}
Background: A large proportion of apprentices shows addictive behaviours like cigarette smoking, alcohol, cannabis, or compulsive Internet use, others do not show such behaviours at all. ready4life is a smartphone application-based coaching program for apprentices, which takes into account the heterogeneity of adolescent addictive behaviour by promoting life skills and reducing risk behaviours. The main objective of the planned study is to test the efficacy of ready4life for addiction prevention among apprentices in Switzerland within a controlled trial.

Methods/design: The efficacy of the ready4life coaching program will be tested in comparison to an assessment only control group, within a cluster-randomised controlled trial with one follow-up assessment after 6 months. At the beginning of the program, participants of the intervention group will receive an individual profile, showing areas in which they have sufficient resources and in which there is a need for coaching. Based on this feedback, they can select two out of the following six program modules: stress, social skills, Internet use, tobacco/e-cigarettes, cannabis, and alcohol. Participants of the intervention group will receive individualised coaching by a conversational agent (chatbot) for a period of four months. The coaching relies on motivational and social-cognitive principles of behaviour change. Within weekly dialogues, the coach provides individually tailored information in different formats, such as videoclips, texts, or pictures. Study participants will be 1318 apprentices with a minimum age of 15, recruited in approximately 100 vocational school classes in Switzerland. Primary outcome will be a composite measure for addictive behaviours including (1) at risk-drinking, (2) tobacco/e-cigarette smoking, (3) cannabis use, and (4) problematic Internet use.
\end{abstract}

Discussion: The study will reveal whether this universally implementable but individually tailored intervention approach is effective in preventing the onset and escalation of addictive behaviors among apprentices.

Trial registration: ISRCTN59908406 (registration date: 21/10/2020).

Keywords: Addiction, Prevention, Apprentices, Coaching, Smartphone

\footnotetext{
* Correspondence: severin.haug@isgf.uzh.ch

Swiss Research Institute for Public Health and Addiction at Zurich University, Konradstrasse 32, 8005 Zurich, Switzerland
}

(c) The Author(s). 2020 Open Access This article is licensed under a Creative Commons Attribution 4.0 International License, which permits use, sharing, adaptation, distribution and reproduction in any medium or format, as long as you give appropriate credit to the original author(s) and the source, provide a link to the Creative Commons licence, and indicate if changes were made. The images or other third party material in this article are included in the article's Creative Commons licence, unless indicated otherwise in a credit line to the material. If material is not included in the article's Creative Commons licence and your intended use is not permitted by statutory regulation or exceeds the permitted use, you will need to obtain permission directly from the copyright holder. To view a copy of this licence, visit http://creativecommons.org/licenses/by/4.0/. The Creative Commons Public Domain Dedication waiver (http://creativecommons.org/publicdomain/zero/1.0/) applies to the data made available in this article, unless otherwise stated in a credit line to the data. 


\section{Background}

Adolescence is characterized by several biological, psychological and social changes, which have a significant impact on the further course of life $[1,2]$. These changes enable greater autonomy, the building of social relationships as well as physical and social identity. However, they are also accompanied by an increased willingness to take risks during a time when the cognitive functions of the brain, e.g. to regulate emotions, are not yet fully developed [3]. This also increases the susceptibility to psychological and substance use disorders [4].

For adolescents starting an apprenticeship, entering work life is accompanied by additional changes and new challenges. Financial independence and social detachment from their parents result in a higher degree of autonomy. At the same time, the responsibility for one's own actions increases and the workplace environment confronts them with the reality of work, which is often accompanied by stress, time and success pressure. Dealing with colleagues, superiors and business partners or customers also places new demands on the social competence of the apprentices. Accordingly, the beginning of the apprenticeship is associated with health risks, especially increased substance consumption. Although corresponding comparative figures from Switzerland are not available, the German drug affinity study shows a significantly higher proportion of tobacco smokers among apprentices than among high school students of the same age (36\% vs. $19 \%)$, whereas the difference is less pronounced for binge drinking, an indicator of problematic alcohol consumption (43\% vs. 39\%) [5]. Apprentices, typically in the age of $15-18$ years, represent a very heterogeneous group in terms of protective and risk behaviours, [6, 7] with a smaller proportion showing no substance use or addictive behaviours and another showing at least one or co-occurring at-risk behaviours. For example in vocational school students from Switzerland, $81 \%$ of smokers showed at-risk alcohol use whereas only $49 \%$ of non-smokers drink hazardously [7].

According to international reviews, general life-skills training programs that address social skills and simultaneously address social influences, e.g. by media or the peer group, are particularly suitable for preventing substance use or delaying the onset of addictive behaviours in younger adolescents and those who have not yet started using addictive substances [8]. In adolescents already using substances, interventions based on motivational and cognitive-behavioural principles proved to be effective in reducing addictive behaviours. They are effective at preventing the onset of specific substance use [9-11] or at decreasing problematic substance use [12]. Due to their age group and their heterogeneous risk profile ranging from none to several co-occurring addictive behaviors, both general life-skills training programs and motivational, cognitive-behavioral programs to reduce specific substances show potential.

Although schools provide access to large numbers of students, the implementation and dissemination of general life-skills or addiction prevention programs in schools pose serious challenges [13]. First, teachers and other professionals need the time, motivation, knowledge and skills to deliver the program. Second, extensive resources in terms of personnel, money, and time are required to administer such programs.

Digital intervention programs, e.g. delivered via computer, Internet or mobile phone have the potential to overcome the above-mentioned obstacles that hinder successful program implementation and larger-scale dissemination of preventive intervention programs in schools. They have a wide reach at a low cost and offer the opportunity to automatically deliver contents that can be accessed at any time and in any place $[14,15]$. Furthermore, they allow an individual tailoring of the intervention program according to the individual health risks, resources and preferences. A meta-analysis on school-based eHealth interventions targeting multiple lifestyle risk behaviours [16] retrieved small and shortterm intervention effects regarding diet, physical activity and screen time, but no effects for alcohol use or smoking behavior, however, no studies of mobile health (mHealth) intervention programs were included.

A promising means of delivering prevention programs is to do so remotely by mobile technologies. In Switzerland, as in most other developed countries, 99\% adolescents between the ages of 12 and 19 own a mobile phone [17], rendering such programs universally implementable. Most adolescents use mobile phones on a daily basis for texting, taking pictures, playing games etc. Mobile phone-based interventions can provide almost constant support to users, in comparison to interventions that can only be accessed at specific times or locations. Mobile phonebased interventions also provide a discrete and confidential means of intervention delivery [18].

Although no mobile phone-delivered interventions addressing multiple lifestyle risk behaviours have been tested yet, mHealth interventions addressing single risk behaviours or general life-skills are promising [6, 19-21]. A controlled trial on a text messaging-based intervention to reduce problem drinking in vocational and upper secondary school students in Switzerland showed a significant intervention effect on the prevalence of binge drinking. For example binge drinking decreased by $6 \%$ in the intervention group and increased by $3 \%$ in the control group, relative to that of baseline assessment [19].

Another Swiss study showed the acceptance and potential effectiveness of a mobile phone-based life-skills training program for substance use prevention among non-smoking vocational school students [6]. Pre-post 
comparisons revealed a decrease in perceived stress and increases in several life skills addressed between baseline and the follow-up assessment. The proportion of adolescents with at-risk alcohol use significantly declined from $20 \%$ at baseline to $15 \%$ at follow-up.

Based on the promising effectiveness of mobile phonedelivered life-skills and addiction prevention programs among vocational school students and the heterogeneous addictive behaviour in this target group of lateadolescents, a reasonable next step is to test the efficacy of a program combining life-skills training and the prevention of multiple specific addictive behaviours within a controlled trial.

Within this study protocol, we describe a cluster randomised controlled trial testing the efficacy of a combined life-skills and addiction prevention coaching program delivered via smartphone to prevent addictive behaviours among apprentices recruited in vocational schools.

\section{Methods/design \\ Design and hypotheses}

We will conduct a two-arm cluster-randomised controlled trial to test the efficacy of ready4life, a smartphone-based life-skills and addiction prevention coaching program to prevent substance use among apprentices. We will test the efficacy of the intervention in comparison to an assessment only control group. The study participants will be assessed at baseline and at 6months follow-up (Fig. 1). Our main hypothesis is that the individually tailored 4-month intervention program will be more effective than assessment only, in preventing the onset and escalation of addictive behaviours including (1) at risk-drinking, (2) tobacco/e-cigarette



Fig. 1 Study design 
smoking, (3) cannabis use, and (4) problematic Internet use at 6-months follow-up.

\section{Participants, setting and procedure}

In most European countries, vocational schools are postsecondary public schools that are analogous to American community colleges. They are part of the dual educational system that combines apprenticeships in a business context and vocational training in a school context. Vocational schools provide general education and specific skills for each particular profession.

Based on data from the Swiss Federal Statistical Office, approximately half of all Swiss adolescents ages 16 to 19 currently attend vocational schools [22], with the highest proportions among adolescents ages 17 (males: 60\%, females: $47 \%$ ) and 18 (males: 57\%, females: 45\%). Vocational schools in the German speaking part of Switzerland will be invited to participate in the study by cooperating regional centres for addiction prevention.

Prevention specialists from these regional centres for addiction prevention will be trained and informed on the ready4life intervention program. These specialists will arrange sessions in the participating vocational school classes, lasting $30 \mathrm{~min}$ during regular school lessons reserved for health education. Within this session, the students will be informed about the program and invited to participate in a study testing innovative channels for the delivery of healthrelated information and life skills. The students will be informed about the study's aims, design, assessments, reimbursement, and data protection. The chatbot coaching program, its friendly competition and the chance to win prizes will be described in detail, using a teaser video (https://www.r4l.swiss/). To ensure sufficient participation and thus representativeness of the sample [23], students will be informed that they would also receive a small reward for participating in the study (10 CHF for completing the follow-up assessment). Participating students will download the app on their smartphone and complete online study registration and baseline assessment. After giving informed consent, study participants will be invited to choose a username and provide their mobile phone number.

Subsequently, participants of intervention classes will receive an individual profile on their risks and resources and can select two topics (stress, social skills, Internet use, tobacco, cannabis, and alcohol) on which they will receive coaching, for a period of four months (two months per topic) by the chatbot. Participants of the assessment only control classes will not receive the intervention program. However, they will be invited to participate in the program after completion of the follow-up assessment in month 6. Follow-up assessment will be conducted in both study groups using a similar procedure: participants will be invited to complete the online follow-up assessments via SMS text messaging.
Non-responders will additionally be addressed via computer-assisted telephone interviews conducted by research assistants.

\section{Ethical review}

The study protocol was approved by the the Ethics Committee of the Faculty of Arts and Sciences at the University of Zurich (approval number 20.10.12; date of approval October, 16th, 2020). The trial will be executed in compliance with the Helsinki Declaration.

\section{Randomisation and allocation concealment}

To avoid spill-over effects within school classes, we will conduct a cluster-randomised controlled trial using school class as a randomisation unit. Due to the heterogeneity of students in the different vocational schools, we will use a separate randomisation list for each school (stratified randomisation). Furthermore, to approximate equality of sample sizes in the study groups, we will use block randomisation with computer generated randomly permuted blocks of 4 cases [24].

The prevention specialists supervising the baseline assessment will be blinded to the group allocation of school classes. In addition, group allocation will not be revealed to participants until they had provided their informed consent, username, mobile phone number, and baseline data. Furthermore, the research assistants who perform the computer-assisted follow up assessments for primary and secondary outcomes will be blinded to the group allocation.

\section{Sample size calculation}

Based on reviews on electronically-delivered programs for the prevention of addictive disorders [25-29], we expect a small effect size for the main outcome of this study, the non-parametric composite measure of addictive behaviours. Based on an expected Cohen's d of 0.2, a sample size of $n=412$ in each study group would be required to have $80 \%$ power for a Wilcoxon-MannWhitney-test ( $\alpha=5 \%, 2$-sided) in order to detect this difference based on a calculation using G-Power. As vocational school students are nested within school classes, we additionally need to consider a potential design effect for the calculation of the sample size for our study. Based on previous efficacy studies in vocational schools $[6,19]$, an average cluster size of 13 study participants per school class and an intra-cluster correlation coefficient of 0.05 could be expected. This would result in a design effect of 1.60. Multiplying this design effect by the required size for an unnested sample $(n=412)$ results in a required sample size of $n=659$ per study group and a total of $n=1318$ study participants. Thus, based on the participation rates of previous efficacy studies among vocational school students [19, 21], 
approximately 100 school classes are required to reach this sample size.

\section{Intervention program}

ready4life (www.r4l.swiss) is a smartphone applicationbased addiction prevention program for adolescents that takes into account the heterogeneity of adolescent addictive behaviour by promoting life skills on the one hand and reducing risk behaviours on the other. The program provides individually tailored coaching by a conversational agent (chatbot, see Fig. 2).

After choosing a male or female avatar, participants are invited by the chatbot to provide demographic data (age and sex) and to complete the baseline assessment on stress, self-efficacy, social skills, Internet, tobacco/ecigarette, cannabis, and alcohol use. Subsequently, an individual feedback is generated on the basis of this survey conducted via smartphone. Using the traffic light colours, this feedback shows areas in which a participant has sufficient resources and in which there is a need for coaching or counselling. Based on this feedback, participants can select two out of the following six program modules: stress, social skills, Internet use, tobacco/e-cigarettes, cannabis, and alcohol. Using the individual data from the baseline assessment, participants receive coaching for a period of 8 weeks for each of the two selected topics. During this 4-month coaching the virtual coach motivates the participants to deal sensibly with addictive substances, gives feedback on current consumption and life skills, and provides individually tailored information in weekly dialogues. Participants are notified of a new module every Tuesday via push message. The time required to process a weekly dialog is between two and five minutes. Participants can also start a conversation with the chatbot themselves by selecting one of several predefined topics within each module (e.g. "quiz on myths about alcohol" within the alcohol module or "using body language effectively" within the module on social skills). In a separate chat within the app ("Ask the Expert") the participants can pose personal questions to regional addiction prevention experts. Young people with high-risk addictive behaviour are encouraged to seek further advice (e.g., regional outpatient counselling services). In order to stimulate active program engagement, several interactive elements such as quiz questions, contests and a playful competition are integrated into ready4life. Program users can collect credits for each completed weekly dialogue with the chatbot. The more credits participants will collect, the higher their chances will be of winning one of several attractive prizes which will be part of a prize draw after program completion. Participants will be able to retrieve their
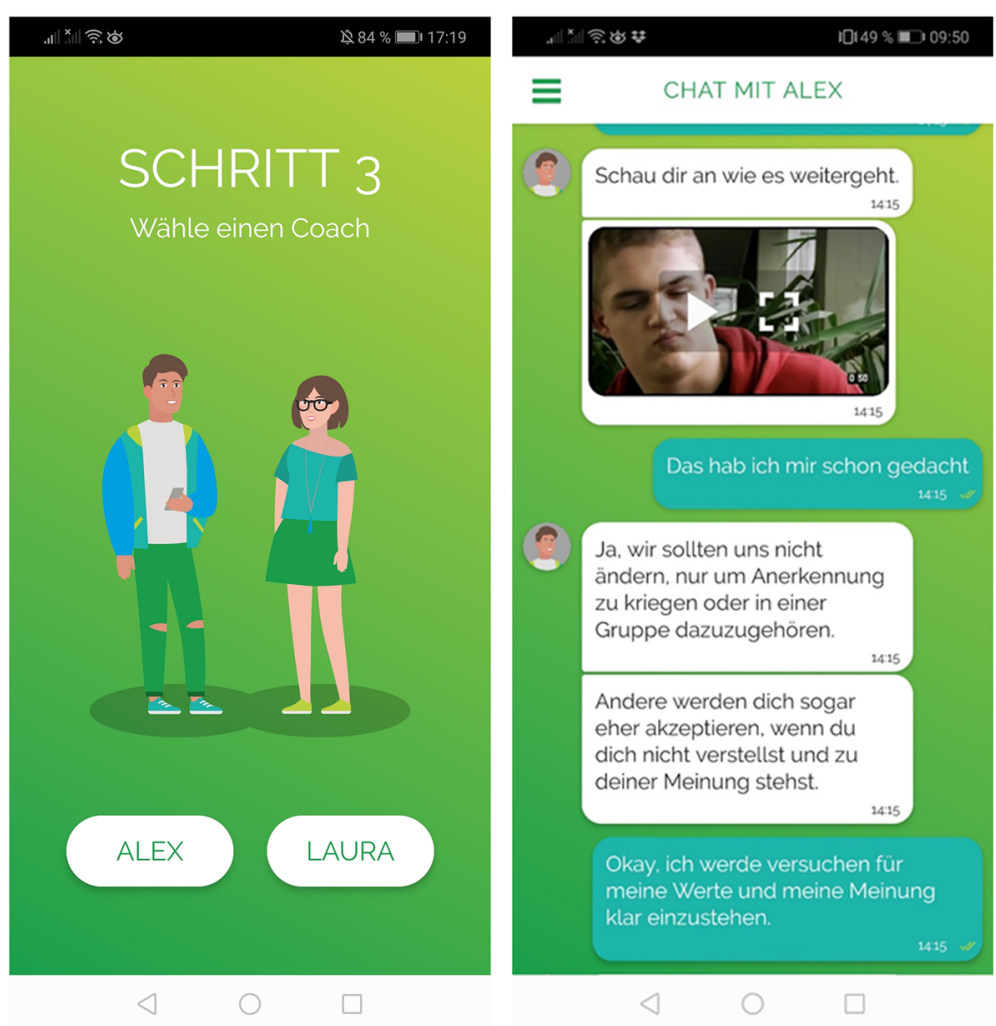

Fig. 2 Screenshots from the ready4life coaching program (left: selection of avatar, right: sample chatbot dialogue) 
Table 1 Coaching contents and elements of ready4life: similar structure for each of the six modules on stress, social skills, Internet use, tobacco/e-cigarettes, cannabis, and alcohol

\begin{tabular}{|c|c|c|c|}
\hline Week & Objective & Example: tobacco/e-cigarettes & Scientific principle \\
\hline 1 & Getting individual feedback & $\begin{array}{l}\text { Feedback on smoking costs } \\
\text { Smoking prevalence in reference group }\end{array}$ & $\begin{array}{l}\text { Self-Monitoring } \\
\text { Normative Feedback }\end{array}$ \\
\hline 2 & $\begin{array}{l}\text { Realizing risks } \\
\text { Motivation to improve skills }\end{array}$ & Pros and cons of smoking and smoking cessation & $\begin{array}{l}\text { Decisional balance } \\
\text { Outcome expectations }\end{array}$ \\
\hline 3 & Learning from other participants & Picture Contest "What could help you or others to become smoke-free?" & $\begin{array}{l}\text { Observational Learning } \\
\text { Facilitation }\end{array}$ \\
\hline 4 & Creating if-then behavior plan & If-then-plan for dealing with temptation in smoking situations & $\begin{array}{l}\text { Implementation Intention } \\
\text { Self-Regulation }\end{array}$ \\
\hline 5 & Clarifying individual questions & Possibility to ask an expert on smoking and smoking cessation & Facilitation \\
\hline 6 & Selecting and pursuing a personal goal & Personal challenge on the subject of smoking & $\begin{array}{l}\text { Goal-setting } \\
\text { Self-Monitoring }\end{array}$ \\
\hline 7 & Learning to deal with difficult situations & $\begin{array}{l}\text { Video quiz on the social norm of smoking in children } \\
\text { Motivation to resist peer pressure }\end{array}$ & $\begin{array}{l}\text { Observational Learning } \\
\text { Self-Efficacy }\end{array}$ \\
\hline 8 & Broadening one's own perspective & Summary of frequently asked questions and expert answers & $\begin{array}{l}\text { Observational Learning } \\
\text { Self-Efficacy }\end{array}$ \\
\hline
\end{tabular}

number of credits compared to the number of credits of other program participants' of their group (similar starting date) at any time from an individual profile page.

Each program module has a similar structure (Table 1) and contains similar intervention elements based on principles of the Social-Cognitive Theory (e.g., goal-setting, self-monitoring, observational learning) [30], the Social Norms Approach (e.g., normative feedback) [31] and Motivational Interviewing (e.g., decisional balance) [32]

\section{Assessments and outcomes}

At baseline, we will assess individual demographic variables (age, sex) as well as characteristics of the schools and school classes (type of vocational school, class size, apprenticeship profession, number of students present within school class). Baseline- and follow-up assessment will include the following addictive behaviours and life skills addressed in the intervention program:

1. At risk-drinking in the preceding 30 days, according to guidelines of the Swiss Federal Office of Public Health [33].

2. 30-days point prevalence for tobacco/e-cigarette smoking, according to the criteria of the Society for Nicotine and Tobacco Research [34].

3. 30-days point prevalence for cannabis use.

4. Problematic Internet use assessed by the Short Compulsive Internet Use Scale [35].

5. General self-efficacy assessed by the Short Scale for Measuring General Self-efficacy Beliefs [36].

6. Stress assessed by a single-item measure of stress symptoms [37].

The primary outcome of the planned study is a composite measure (range: 0-4) for addictive behaviours, reflecting the number of risk behaviours (at-risk drinking, tobacco/e-cigarette smoking, cannabis use, problematic Internet use) that a person displays. Secondary outcomes are metric measures reflecting (1) alcohol use, (2) tobacco/e-cigarette use, (3) cannabis use, (4) problematic Internet use as well as (5) general self-efficacy [36] and (6) stress [37].

\section{Data analyses}

Generalized Linear Mixed Models will be used to test intervention effects for binary and count outcomes and Linear Mixed Models for continuous outcomes [38, 39]. These models account for both fixed and random effects and are particularly useful in analysing longitudinal and nested data (e.g., students within school classes). To test the efficacy of the intervention, we will test the variables "study group", "time" and their interaction "study group $x$ time" as predictors of the outcome criteria assessed at follow-up. If necessary, we will control baseline differences by adding additional baseline variables as covariates to the models. We will conduct both (1) intention to treat analyses and (2) complete case analyses considering all study participants with available follow-up data. For ITT analyses, we will use multiple imputation procedures as described elsewhere [40].

\section{Discussion}

Entering work life, typically in late adolescence, is associated with mutual challenges and health risks. ready4life is the first mHealth program for addiction prevention in apprentices that will be tested within a controlled trial. The program takes into account the heterogeneity of adolescent addictive behaviour by promoting life skills on the one hand and reducing risk behaviours on the other. Addressing four major addictive behaviours 
among adolescents [8] as well as two life-skills does not only have the potential to reduce substance and internet use in the short term, but may also lead to increased general self-efficacy and life skills, such as coping with stress, which in the long term might help to prevent further non-communicable diseases and improve wellbeing $[41,42]$. In contrast to comprehensive schoolbased curricula, training and counselling via the ready4life smartphone app is more economic and corresponds with the lifestyle and communication habits of the target group. A majority of apprentices are familiar with how to use smartphones and typically use them on a daily basis for texting, taking photos, playing games etc. Nonetheless, continued use and adherence to eHealth or mHealth intervention programs presents a serious challenge $[43,44]$. Although, we implemented several measures like push notifications, gamification elements like quizzes, the collection of credits for program engagement or a price draw, we are aware that the app will compete with many others. Therefore, besides program efficacy, the investigation of program engagement and its association with program outcome presents another focus of this research project $[43,45]$.

The presented efficacy study on this program is of high scientific and public health relevance. It will reveal whether this universally implementable but individually tailored intervention approach is effective in increasing life skills and reducing risk behaviours in a group of adolescents with a particular high risk of addictive behaviours [7]. If this program proves to be effective, it could be disseminated to apprentices in different settings, e.g., schools, companies or via online platforms for apprentices or vocational students. Furthermore, an adaption for youth in general or the translation of the intervention content into other languages would easily enable program dissemination.

\section{Acknowledgements}

We would like to thank Sarah Eschmann and her team from the Swiss Lung Association for supporting this controlled study. Furthermore, we would like to thank Andreas Filler and his team from PathMate Technologies for their technical assistance for this project.

\section{Authors' contributions}

SH and RPC were responsible for the study design. SH, RPC, MPS and AW developed the contents and flow of the smartphone-based intervention program. SH, AW and RPC are responsible for the data collection. All authors read and approved the final manuscript.

\section{Funding}

Funding for this study is provided by the Research Fund of the Swiss Lung Association. The funder has no role in study design, data collection and analysis, decision to publish or preparation of manuscripts.

\section{Availability of data and materials}

The datasets generated during the current study are available from the corresponding author on reasonable request.

\section{Ethics approval and consent to participate}

The study protocol was approved by the the Ethics Committee of the Faculty of Arts and Sciences at the University of Zurich (approval number 20.10.12; date of approval October, 16th, 2020). Informed consent will be obtained online from all study participants within the study registration procedure of the ready4life smartphone application.

\section{Consent for publication}

Not applicable.

\section{Competing interests}

The authors declare that they have no competing interests.

Received: 10 November 2020 Accepted: 30 November 2020

Published online: 14 December 2020

\section{References}

1. Costello EJ, Copeland W, Angold A. Trends in psychopathology across the adolescent years: what changes when children become adolescents, and when adolescents become adults? J Child Psychol Psychiatry. 2011;52(10): 1015-25.

2. Hall WD, Patton G, Stockings E, Weier M, Lynskey M, Morley Kl, Degenhardt $L$. Why young people's substance use matters for global health. Lancet Psychiatry. 2016;3(3):265-79.

3. White AM. Understanding adolescent brain development and its implications for the clinician. Adolesc Med State Art Rev. 2009:20(1):73-90 viii-ix.

4. Gore FM, Bloem PJ, Patton GC, Ferguson J, Joseph V, Coffey C, Sawyer SM, Mathers CD. Global burden of disease in young people aged 10-24 years: a systematic analysis. Lancet. 2011;377(9783):2093-102.

5. Orth B: Die Drogenaffinität Jugendlicher in der Bundesrepublik Deutschland 2015. Rauchen, Alkoholkonsum und Konsum illegaler Drogen: aktuelle Verbreitung und trends. BZgA-Forschungsbericht [the drug affinity of young people in Germany 2015. Smoking, alcohol consumption and use of illegal drugs: current prevalence and trends. BZgA research report]. Köln: Bundeszentrale für gesundheitliche Aufklärung; 2016.

6. Haug S, Paz Castro R, Meyer C, Filler A, Kowatsch T, Schaub MP. A mobile phone-based life-skills training for substance use prevention among adolescents: a pre-post study on the acceptance and potential effectiveness of the program Ready4life. J Med Internet Res mHealth uHealth. 2017:5(9):e143.

7. Haug S, Schaub MP, Salis Gross C, John U, Meyer C. Predictors of hazardous drinking, tobacco smoking and physical inactivity in vocational school students. BMC Public Health. 2013;13:475.

8. Stockings E, Hall WD, Lynskey M, Morley KI, Reavley N, Strang J, Patton G, Degenhardt L. Prevention, early intervention, harm reduction, and treatment of substance use in young people. Lancet Psychiatry. 2016;3(3):280-96.

9. Weichold K, Blumenthal A. Long-term effects of the life skills program IPSY on substance use: results of a 4.5-year longitudinal study. Prev Sci. 2016; 17(1):13-23.

10. Velasco V, Griffin KW, Botvin GJ, Celata C, Lombardia GL. Preventing adolescent substance use through an evidence-based program: effects of the Italian adaptation of life skills training. Prev Sci. 2017;18(4):394-405.

11. Thomas RE, McLellan J, Perera R. School-based programmes for preventing smoking. Cochrane Database Syst Rev. 2013;4:CD001293.

12. Foxcroft DR, Tsertsvadze A. Universal school-based prevention programs for alcohol misuse in young people. Cochrane Database Syst Rev. 2011;5: CD009113.

13. Ellickson PL. You've shown the program is effective. Now what? In: Weichold K, Giannotta F, editors. Theory-Based Approaches to Substance Misuse and Abuse Prevention in School. San Francisco: Jossey-Bass; 2014. p. 95-106.

14. Kuntsche $E$, Labhart $F$. The future is now-using personal cellphones to gather data on substance use and related factors. Addiction. 2014;109(7): 1052-3.

15. Naughton F. Delivering "just-in-time" smoking cessation support via Mobile phones: current knowledge and future directions. Nicotine Tob Res. 2017; 19(3):379-83.

16. Champion KE, Parmenter B, McGowan C, Spring B, Wafford QE, Gardner LA, Thornton L, McBride N, Barrett EL, Teesson M, et al. Effectiveness of schoolbased eHealth interventions to prevent multiple lifestyle risk behaviours among adolescents: a systematic review and meta-analysis. Lancet Digit Health. 2019;1(5):E206-21. 
17. Suter L, Waller G, Bernath J, Külling C, Willemse I, Süss D. JAMES: Jugend Aktivitäten, Medien - Erhebung Schweiz [JAMES: Youth, Activity, Media Data Switzerland]. Zurich: Zürcher Hochschule für angewandte Wissenschaften; 2018.

18. Kenny R, Dooley B, Fitzgerald A. Feasibility of "CopeSmart": a Telemental health app for adolescents. JMIR Ment Health. 2015;2(3):e22.

19. Haug S, Paz Castro R, Kowatsch T, Filler A, Dey M, Schaub MP. Efficacy of a web- and text messaging-based intervention to reduce problem drinking in adolescents: results of a cluster-randomized controlled trial. J Consult Clin Psychol. 2017;85(2):147-59.

20. Haug S, Paz Castro R, Scholz U, Kowatsch T, Schaub MP, Radtke T. Assessment of the efficacy of a Mobile phone-delivered just-in-time planning intervention to reduce alcohol use in adolescents: randomized controlled crossover trial. JMIR Mhealth Uhealth. 2020;8(5):e16937.

21. Haug S, Schaub MP, Venzin V, Meyer C, John U. Efficacy of a text messagebased smoking cessation intervention for young people: a cluster randomized controlled trial. J Med Internet Res. 2013;15(8):e171.

22. Bundesamt für Statistik: Schulbesuchsquoten der 16-26-Jährigen 2018/19 [School attendance rates among 16-26 year olds in 2018/19]. Neuchâtel: Bundesamt für Statistik; 2020.

23. Edwards P, Cooper R, Roberts I, Frost C. Meta-analysis of randomised trials of monetary incentives and response to mailed questionnaires. J Epidemiol Community Health. 2005:59(11):987-99.

24. Pocock SJ. Clinical trials: a practical approach. Chichester: Wiley \& Sons; 1994.

25. Kaner EF, Beyer FR, Garnett C, Crane D, Brown J, Muirhead C, Redmore J, O'Donnell A, Newham JJ, de Vocht F, et al. Personalised digital interventions for reducing hazardous and harmful alcohol consumption in communitydwelling populations. Cochrane Database Syst Rev (Online). 2017;9:CD011479.

26. Kazemi DM, Borsari B, Levine MJ, Li S, Lamberson KA, Matta LA. A systematic review of the mHealth interventions to prevent alcohol and substance abuse. J Health Commun. 2017;22(5):413-32.

27. Oosterveen E, Tzelepis F, Ashton L, Hutchesson MJ. A systematic review of eHealth behavioral interventions targeting smoking, nutrition, alcohol, physical activity and/or obesity for young adults. Prev Med. 2017:99:197-206.

28. Teesson M, Newton NC, Slade T, Carragher N, Barrett EL, Champion KE, Kelly EV, Nair NK, Stapinski LA, Conrod PJ. Combined universal and selective prevention for adolescent alcohol use: a cluster randomized controlled trial. Psychol Med. 2017:47(10):1761-70.

29. Whittaker R, McRobbie H, Bullen C, Rodgers A, Gu Y, Dobson R. Mobile phone text messaging and app-based interventions for smoking cessation. Cochrane Database Syst Rev (Online). 2019;10:CD006611.

30. McAlister AL, Perry CL, Parcel GS. How individuals, environments, and health behaviour interact: social cognitive theory. In: Glanz K, Rimer BK, Viswanath K, editors. Health Behavior and Health Education: Theory, Research, and Practice. San Francisco: Jossey-Bass; 2008.

31. Perkins HW. The social norms approach to preventing school and college age substance abuse: a handbook for educators, counselors, and clinicians., first edn. San Francisco: Jossey-Bass; 2003.

32. Miller WR, Rollnick S. Motivational interviewing: helping people change, 3rd edition. New York: Guilford Press; 2013.

33. Eidgenössische Kommission für Alkoholfragen EKAL: Orientierungshilfe zum Alkoholkonsum [Orientation guide to alcohol consumption]. Bern: Eidgenössische Kommission für Alkoholfragen; 2018.

34. Hughes JR, Keely JP, Niaura RS, Ossip-Klein DJ, Richmond RL, Swan GE. Measures of abstinence in clinical trials: issues and recommendations. Nicotine Tob Res. 2003;5(1):13-25.

35. Besser B, Rumpf HJ, Bischof A, Meerkerk GJ, Higuchi S, Bischof G. Internetrelated disorders: development of the short compulsive internet use scale. Cyberpsychol Behav Soc Netw. 2017;20(11):709-17.

36. Beierlein C, Kovaleva A, Kemper CJ, Rammstedt B. Ein Messinstrument zur Erfasssung subjektiver Kompetenzerwartungen. Allgemeine Selbstwirksamkeit Kurzskala (ASKU) [A Short Scale for Measuring General Self-efficacy Beliefs (ASKU)]. Köln: GESIS; 2012.

37. Elo AL, Leppanen A, Jahkola A. Validity of a single-item measure of stress symptoms. Scand J Work Environ Health. 2003;29(6):444-51.

38. Laird NM, Ware JH. Random-effects models for longitudinal data. Biometrics. 1982;38(4):963-74.

39. Twisk JW. Applied longitudinal data analysis for epidemiology: a practical guide. Cambridge: University Press; 2013.

40. Van Buuren S. Flexible imputation of missing data. Boca Raton: CRC Press; 2012.
41. Endrighi R, Dimond AJ, Waters AJ, Dimond CC, Harris KM, Gottlieb SS, Krantz DS. Associations of perceived stress and state anger with symptom burden and functional status in patients with heart failure. Psychol Health. 2019; 34(10):1250-66.

42. Mazor M, Paul SM, Chesney MA, Chen LM, Smoot B, Topp K, Conley YP, Levine JD, Miaskowski C. Perceived stress is associated with a higher symptom burden in cancer survivors. Cancer. 2019;125(24):4509-15.

43. Sieverink F, Kelders SM, van Gemert-Pijnen JE. Clarifying the concept of adherence to eHealth technology: systematic review on when usage becomes adherence. J Med Internet Res. 2017;19(12):e402.

44. Deady M, Glozier N, Collins D, Einboden R, Lavender I, Wray A, Gayed A, Calvo RA, Christensen $\mathrm{H}$, Harvey SB, et al. The utility of a mental health app in apprentice workers: a pilot study. Front Public Health. 2020:8:389.

45. Paz Castro R, Haug S, Filler A, Kowatsch T, Schaub MP. Engagement within a Mobile phone-based smoking cessation intervention for adolescents and its association with participant characteristics and outcomes. J Med Internet Res. 2017;19(11):e356.

\section{Publisher's Note}

Springer Nature remains neutral with regard to jurisdictional claims in published maps and institutional affiliations.
Ready to submit your research? Choose BMC and benefit from:

- fast, convenient online submission

- thorough peer review by experienced researchers in your field

- rapid publication on acceptance

- support for research data, including large and complex data types

- gold Open Access which fosters wider collaboration and increased citations

- maximum visibility for your research: over $100 \mathrm{M}$ website views per year

At BMC, research is always in progress.

Learn more biomedcentral.com/submissions 